\title{
Retreatment of vestibular schwannoma with Gamma Knife radiosurgery: clinical outcome, tumor control, and review of literature
}

\author{
Victor X. Fu, MD, ${ }^{1}$ Jeroen B. Verheul, MD, PhD, ${ }^{1}$ Guus N. Beute, MD, ${ }^{1}$ Sieger Leenstra, MD, PhD, ${ }^{1}$ \\ Henricus P. M. Kunst, MD, PhD, ${ }^{2}$ Jef J. S. Mulder, MD, PhD, ${ }^{2}$ and Patrick E. J. Hanssens, MD ${ }^{1}$ \\ ${ }^{1}$ Gamma Knife Center Tilburg, Department of Neurosurgery, St. Elisabeth Hospital, Tilburg; and ${ }^{2 D e p a r t m e n t ~ o f ~ E N T, ~ R a d b o u d ~}$ \\ University Medical Center, Nijmegen, The Netherlands
}

\begin{abstract}
OBJECTIVE Gamma Knife radiosurgery (GKRS) has become an accepted treatment for vestibular schwannoma, with a high rate of tumor control and good clinical outcome. In a small number of cases, additional treatment is needed. This retrospective study examines the clinical outcome, reproducibility of volumetric response patterns, and tumor control rate after administering a second GKRS to treat vestibular schwannomas.
\end{abstract}

METHODS A total of 38 patients were included: 28 patients underwent a radiosurgical procedure as the initial treatment (Group 1), and 10 patients underwent microsurgical resection with adjuvant radiosurgery on the tumor remnant as the initial treatment (Group 2). The indication for a second GKRS treatment was growth observed on follow-up imaging. The median margin dose was $11.0 \mathrm{~Gy}$ for the first procedure and $11.5 \mathrm{~Gy}$ for the second procedure. Tumor control after retreatment was assessed through volumetric analysis. Clinical outcome was assessed through medical chart review.

RESULTS Median tumor volume at retreatment was $3.6 \mathrm{~cm}^{3}$, with a median treatment interval of 49 months. All patients showed tumor control in a median follow-up period of 75 months after the second radiosurgical procedure. Volumetric tumor response after the second procedure did not correspond to response after the first procedure. After retreatment, persisting House-Brackmann Grade II facial nerve dysfunction was observed in 3 patients (7.9\%), facial spasms in 5 patients $(13 \%)$, and trigeminal nerve hypesthesia in 3 patients $(7.9 \%)$. Hearing preservation was not evaluated because of the small number of patients with serviceable hearing at the second procedure.

CONCLUSIONS Repeat GKRS after a failed first treatment appears to be an effective strategy in terms of tumor control. The volumetric response after a repeat procedure could not be predicted by the volumetric response observed after first treatment. This justifies considering repeat GKRS even for tumors that do not show any volumetric response and show continuous growth after first treatment. An increased risk of mild facial and trigeminal nerve dysfunction was observed after the second treatment compared with the first treatment.

https://thejns.org/doi/abs/10.3171/2017.3.JNS162033

KEY WORDS vestibular schwannoma; acoustic neuroma; Gamma Knife; retreatment; tumor control; stereotactic radiosurgery

$\mathrm{G}$ AMMA Knife radiosurgery (GKRS) is an accepted treatment for vestibular schwannoma, with reported 5- to 10-year success rates of over 93\%, good quality of life, and preservation of function., ${ }^{5,10,15}$ Also, stereotactic radiosurgery is an alternative to additional microsurgery for patients in whom prior microsurgical tumor removal has failed or as an adjuvant treatment after planned subtotal resection. ${ }^{17,21}$ However, in a small number of cases, retreatment of vestibular schwannoma is needed because of either continuous growth progression or regrowth of the tumor seen after initial tumor control during follow-up. Whether this second procedure should be microsurgery or radiosurgery remains debatable, ${ }^{16,18,19}$ especially in patients in whom no volumetric tumor response was shown after radiosurgical treatment. This retrospective study examines clinical outcome and tumor control after a second GKRS procedure (GKRS 2) in patients with vestibular schwannoma tumors. Furthermore,

ABBREVIATIONS GKRS = Gamma Knife radiosurgery; GKRS 1 = first GKRS procedure; GKRS 2 = second GKRS procedure.

SUBMITTED August 4, 2016. ACCEPTED March 6, 2017.

INCLUDE WHEN CITING Published online October 6, 2017; DOI: 10.3171/2017.3.JNS162033. 
the volumetric response patterns after the first GKRS procedure (GKRS 1) and GKRS 2 were compared to evaluate whether the volumetric response after GKRS 2 could be predicted by the response to GKRS 1. Finally, a literature search was performed to review the current results and opinions on GKRS retreatment.

\section{Methods \\ Patient Inclusion}

Between June 10, 2002, and December 1, 2016, 1156 patients underwent GKRS as treatment for unilateral vestibular schwannoma. The main treatment indication was a small- to medium-size vestibular schwannoma with documented growth, a large tumor at initial presentation in which case a wait-and-scan strategy was not appropriate, or declining serviceable hearing. Generally, patients with tumors larger than $30 \mathrm{~mm}$ in diameter on MRI were treated with radiosurgery when microsurgical resection was contraindicated because of significant comorbidity or advanced age. Patient follow-up included assessment of facial and trigeminal nerve function and MRI with a standard interval of 1 year, which could be shortened or extended based on individual indications, prognosis, and tumor size. Subjective changes in hearing level were evaluated, and audiometry was performed when indicated. The decision for additional treatment, either repeat GKRS or microsurgical resection, was made primarily based on linear (2D) tumor growth during annual follow-up with MRI and discussed in a multidisciplinary session. Transient radiation-induced tumor swelling was taken into account during the first 2 years after radiosurgical treatment. When it was concluded that GKRS 1 had failed, GKRS 2 was advised with similar treatment indications as GKRS 1. All GKRS retreatments were done within 3 months of assessed treatment failure. A total of 18 patients with treatment failure after GKRS 1 underwent microsurgical resection, and 63 patients underwent GKRS 2. Patients with less than 1 year of follow-up and no MRI scans ( 3 patients), neurofibromatosis Type 2 (8 patients), resection between GKRS treatments ( 9 patients), or incomplete data (5 patients) were excluded. Finally, 38 patients were included (Fig. 1), with 28 patients undergoing primary GKRS retreatment (Group 1) and 10 patients receiving GKRS 2 to treat the tumor remnant after failed initial microsurgical resection and adjuvant GKRS (Group 2). The indication for primary resection in Group 2 was diverse because a substantial number of patients had their tumors resected at other centers and were referred to our center for a radiosurgical procedure. The microsurgical strategy varied because some centers aimed for a planned subtotal resection, while others for gross total resection. Also, the decision to refer patients for radiosurgery to treat the tumor remnant was either made immediately after surgery or delayed and performed only after growth of the tumor remnant was observed.

\section{GKRS Treatment Protocol}

Until November 2008, the Leksell Gamma Knife model 4C was used, while from November 2008 onward the Leksell Gamma Knife Perfexion model was used.
T1-weighted MRI scans both with and without gadolinium $(0.1 \mathrm{mg} / \mathrm{kg}$ body weight), as well as T2-weighted MRI scans, were obtained for dose planning. Until May 2011, a radiation dose of 13.0 Gy was prescribed to the isodose covering $90 \%$ of the tumor volume, assuring a margin dose of 11.0 Gy to $100 \%$ of the tumor volume. Since May 2011, a dose of 12.5-13.0 Gy has been prescribed to 99\% of the tumor volume. These dose prescription protocols were used in both GKRS 1 and GKRS 2. Dose calculations were performed with GammaPlan Wizard (version 5.32) or Leksell GammaPlan 4C prior to November 2008. Since November 2008, Leksell GammaPlan (version 8.3.1 or 9.0.0) has been used for the dose calculation for the Perfexion model.

\section{Data Analysis}

To analyze tumor control and the tumor growth patterns after GKRS, the tumor volumes were measured for all treatment plans and follow-up MR images through December 1, 2016. Tumor volume was calculated using an algorithm after hand-drawing the tumor contours on the axial, coronal, and sagittal MR images using SmartBrush p2.0.5 (Brainlab Element). Tumor control after GKRS 2 meant either significant regression of tumor volume, stable tumor volume, or transient expansion. We defined regression as tumor volume reduction of at least $10 \%$, stable as less than $10 \%$ reduction in tumor volume, and enlargement as tumor volume enlargement more than $10 \%$. In cases in which there was volumetric enlargement exceeding $10 \%$ but also in which we observed a significant regression of tumor volume on the last MR image compared with the peak tumor volume seen after treatment, the tumor growth pattern was reported as transient expansion. To assess possible predictors of tumor enlargement after retreatment, univariate and multivariate binary logistic regression analyses were used to assess the following parameters: volume response pattern after GKRS 1, tumor volume at retreatment, treatment interval between both GKRS procedures, prior microsurgical resection, and tumor margin radiation dose. Patient charts were reviewed for clinical complications, which were routinely assessed during follow-up interviews and clinical examinations by a neurosurgeon or radiation oncologist.

\section{Literature Search}

A literature search on PubMed was performed using the following query terms: "gamma knife" [All Fields] OR "stereotactic radiosurgery" [All Fields] AND "acoustic neuroma" [All Fields] OR "vestibular schwannoma" [All Fields] AND ("retreatment" [All Fields] OR "repeat" [All Fields]).

Articles were included if the study contained patients who had undergone repeat radiosurgical treatment of vestibular schwannoma when tumor control was not achieved after primary radiosurgical treatment. Articles were excluded if we could not achieve access to the full text. Case reports were also excluded. The relevance of the resulting articles was assessed by reading the titles and abstracts. Also, cross-checking of the references for relevant articles was performed. 


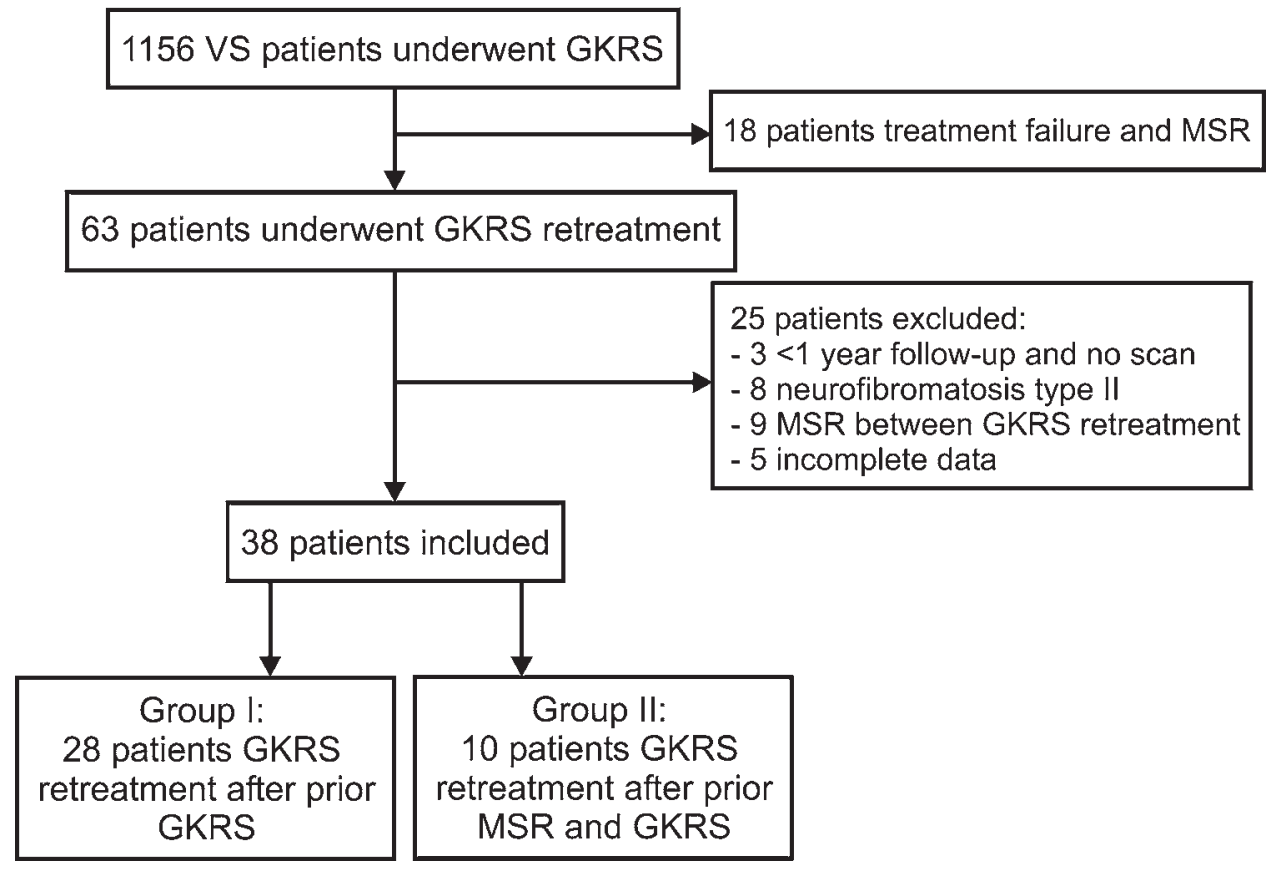

FIG. 1. Patient inclusion flow chart. MSR = microsurgical resection; VS = vestibular schwannoma.
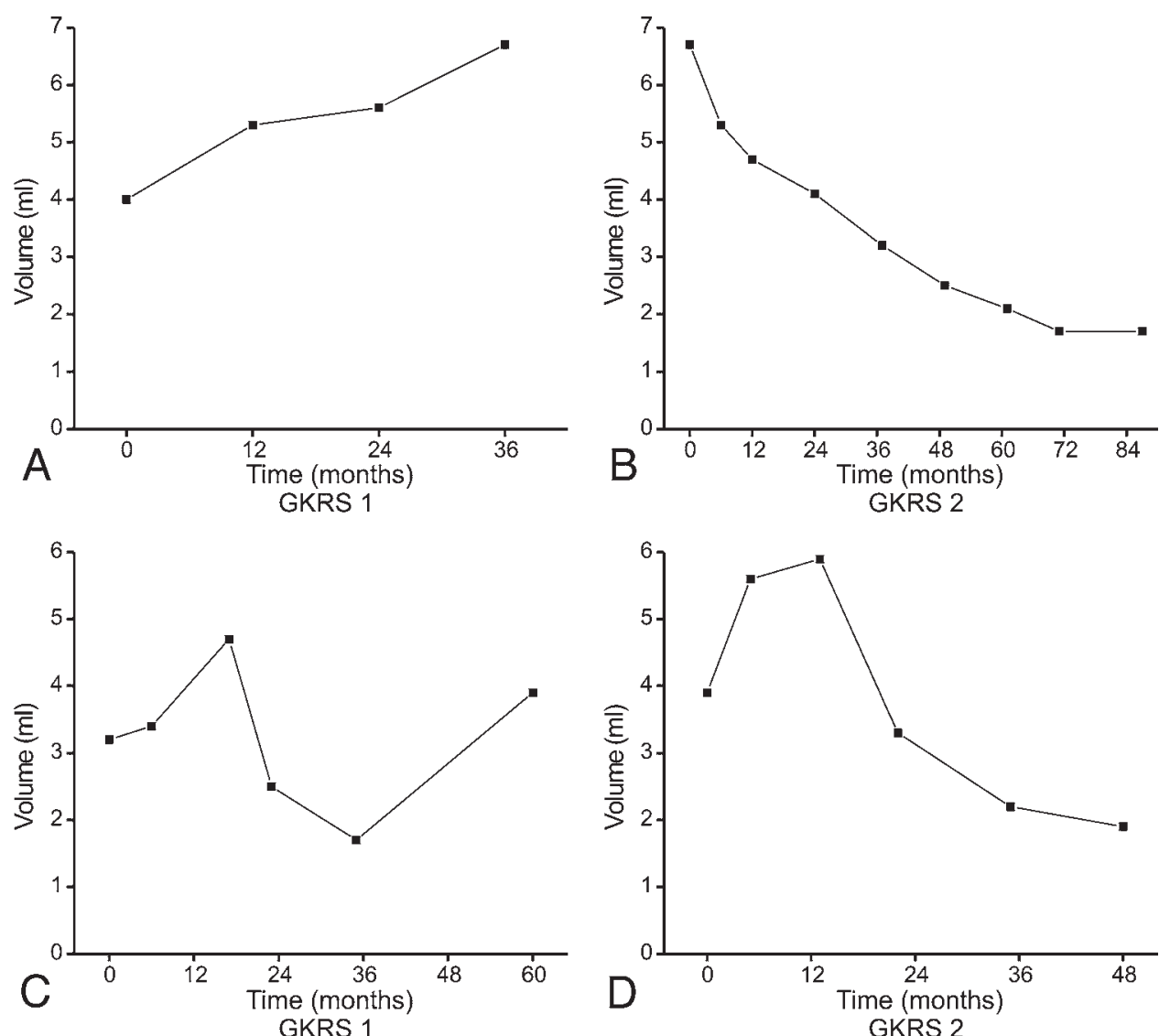

FIG. 2. Examples of volumetric response patterns. Graphs of 2 patients after GKRS 1 and GKRS 2. Time 0 indicates the time of treatment. Continuous growth $(\mathbf{A})$, direct regression after retreatment $(\mathbf{B})$, transient expansion and regrowth $(\mathbf{C})$, and transient expansion and regression after retreatment (D) are shown. Note the peak transient expansion volume at 12 months. 


\section{Results}

Tumor control after retreatment was achieved in all 38 patients. All patients were alive at the last follow-up.

\section{Group 1}

Twenty-eight patients with vestibular schwannoma were treated with GKRS and re-treated with GKRS 2 because they either showed continuous tumor growth (13 patients) or tumor regrowth after the initial treatment response to GKRS 1 (15 patients) (see Fig. 2 for examples). The patient characteristics of Group 1 are shown in Table 1 , tumor volume and treatment dose are shown in Table 2, and neurological outcome is shown in Table 3. Median tumor volume was $1.7 \mathrm{~cm}^{3}$ at GKRS 1 and $2.9 \mathrm{~cm}^{3}$ at GKRS 2 , with a median treatment interval of 52 months between GKRS procedures. Median tumor volume at the last follow-up was $2.1 \mathrm{~cm}^{3}$ for Group 1 with a median follow-up period after retreatment of 66 months. After retreatment, 19 patients had tumor regression at the last follow-up, of which 12 patients $(43 \%)$ had direct tumor regression and 7 patients $(25 \%)$ had regression after transient expansion. Five patients (18\%) had stabilization of the tumor at the last follow-up. A total of 4 patients had a larger tumor volume at the last follow-up, of whom 2 patients (7.1\%) had a transient expansion pattern without reaching regression and 2 patients had enlargement with stabilization of tumor volume on the last 3 consecutive follow-up MRI scans (7.1\%). In 12 of 28 patients (46\%), transient expansion was observed.

During the follow-up period after GKRS 2, 4 patients developed House-Brackmann Grade II facial nerve dysfunction, which seems to be permanent in 2 patients (7.1\%). New facial spasms after GKRS 2 were observed in 9 patients (32\%), which in 5 cases seem to be permanent $(18 \%)$. In all cases, these spasms occurred within 6 to 18 months after GKRS 2, and spasms lasted 6 to 12 months in 4 patients. In 1 patient with facial spasms after GKRS 1, these spasms did not change after GKRS 2. New trigeminal nerve hypesthesia after GKRS 2 was reported in 4 patients, with 2 patients regaining full sensibility (7.1\%). In 2 patients with trigeminal nerve hypesthesia after GKRS 1 , trigeminal nerve function did not worsen after GKRS 2. One patient developed a symptomatic adverse radiation effect and had to be hospitalized because of edema in the cerebellar peduncle, which was successfully treated with dexamethasone and mannitol. Neurological outcome was not consistent with volumetric response after retreatment, and in some cases neurological symptoms developed while tumor volume decreased. Two patients had functional hearing prior to retreatment, and 6 patients had an unspecified degree of hearing. In 2 patients, hearing decreased subjectively, which was confirmed with audiometry in 1 patient.

\section{Group 2}

Ten patients with vestibular schwannoma who underwent GKRS 2 had initially been treated with a microsurgical resection followed by GKRS on the tumor remnant. The patient characteristics of Group 2 are shown in Table 4, tumor volume and treatment dose are shown in Table 5,
TABLE 1. Patient characteristics of Group 1

\begin{tabular}{|c|c|}
\hline Characteristic & Value \\
\hline No. of patients & 28 \\
\hline Mean age (range) at GKRS 2, yrs & $57(42-81)$ \\
\hline \multicolumn{2}{|l|}{ Sex } \\
\hline Male & 15 \\
\hline Female & 13 \\
\hline \multicolumn{2}{|l|}{ Side of body } \\
\hline Right & 13 \\
\hline Left & 15 \\
\hline Median interval btwn treatments, mos & $52(19-86)$ \\
\hline Median follow-up (range), mos & $66(13-129)$ \\
\hline Median margin dose for GKRS 1 (range), Gy & $11.0(10.4-12.3)$ \\
\hline Median margin dose for GKRS 2 (range), Gy & $11.8(9.80-13.0)$ \\
\hline Median tumor volume at GKRS 1 (range), $\mathrm{cm}^{3}$ & $1.7(0.072-7.8)$ \\
\hline Median tumor volume at GKRS 2 (range), $\mathrm{cm}^{3}$ & $2.9(0.27-9.4)$ \\
\hline Median tumor volume at last follow-up (range), $\mathrm{cm}^{3}$ & $2.1(0.10-9.4)$ \\
\hline \multicolumn{2}{|l|}{ Growth pattern after GKRS 1} \\
\hline Regrowth & 15 \\
\hline Continuous & 13 \\
\hline Transient expansion after GKRS 2 & 12 \\
\hline \multicolumn{2}{|l|}{ Retreatment result at last follow-up } \\
\hline Regression & 19 \\
\hline Stable & 5 \\
\hline Transient expansion & 2 \\
\hline Stable enlargement & 2 \\
\hline
\end{tabular}

and neurological outcome is shown in Table 6. Median tumor volume was $1.6 \mathrm{~cm}^{3}$ at GKRS 1 and $3.9 \mathrm{~cm}^{3}$ at GKRS 2 , with a median interval between GKRS procedures of 35 months. Median tumor volume in Group 2 at last followup was $2.1 \mathrm{~cm}^{3}$, with a median follow-up after retreatment of 94 months. In 7 patients (70\%), direct tumor regression was achieved after retreatment, 2 patients (20\%) had regression after transient expansion, and 1 patient (10\%) had a stable tumor volume.

Three patients had mild, House-Brackmann Grade II facial nerve dysfunction after resection and radiosurgery of the tumor remnant prior to GKRS retreatment, which did not worsen in 2 patients. In 1 patient, House-Brackmann Grade II facial nerve dysfunction temporarily worsened to House-Brackmann Grade IV, while a patient without previous dysfunction developed House-Brackmann Grade III function, which lessened over time to Grade II. No patient in Group 2 had functional hearing prior to GKRS 2.

\section{Statistical Analysis}

Volume response pattern after the GKRS 1 (OR 0.63; $\mathrm{p}=0.60)$, tumor volume at retreatment (OR 1.1; $\mathrm{p}=0.62)$, treatment interval $(\mathrm{OR} 1.0 ; \mathrm{p}=0.77)$, previous microsurgical resection (corrected for tumor volume) (OR $0.23 ; \mathrm{p}=$ $0.10)$, and margin dose (OR 1.4; $\mathrm{p}=0.54$ ) were not statistically significant predictors of transient enlargement after retreatment. No statistical analysis was performed to ana- 
TABLE 2. Tumor volumetric responses of Group 1

\begin{tabular}{|c|c|c|c|c|c|c|c|c|c|}
\hline Case No. & TV1 & MD1 & Growth Pattern 1* & INT & TV2 & MD2 & $\mathrm{FU}$ & TV3 & Growth Pattern $2 \dagger$ \\
\hline 1 & 0.9 & 11.1 & Regrowth & 50 & 1.6 & 12.4 & 57 & 1.1 & Regression \\
\hline 2 & 4.2 & 11.0 & Continuous & 31 & 5.7 & 11.8 & 62 & 8.7 & Enlargement, stable \\
\hline 3 & 0.7 & 10.7 & Regrowth & 58 & 1.0 & 12.4 & 70 & 0.5 & Regression \\
\hline 4 & 6.6 & 10.4 & Regrowth & 29 & 6.3 & 11.6 & 72 & 3.0 & Transient expansion, regression \\
\hline 5 & 0.9 & 10.5 & Regrowth & 58 & 1.6 & 11.0 & 74 & 1.0 & Regression \\
\hline 6 & 2.4 & 11.0 & Continuous & 19 & 4.7 & 11.2 & 75 & 4.2 & Transient expansion, regression \\
\hline 7 & 1.7 & 11.5 & Regrowth & 62 & 2.8 & 11.2 & 82 & 0.8 & Regression \\
\hline 8 & 5.1 & 10.7 & Regrowth & 48 & 4.7 & 11.5 & 89 & 2.5 & Regression \\
\hline 9 & 5.9 & 11.3 & Regrowth & 30 & 9.4 & 11.2 & 95 & 2.3 & Regression \\
\hline 10 & 1.1 & 10.5 & Continuous & 26 & 2.8 & 11.3 & 100 & 2.3 & Transient expansion, regression \\
\hline 11 & 1.6 & 10.5 & Continuous & 37 & 3.8 & 11.2 & 48 & 3.6 & Transient expansion, stable \\
\hline 12 & 0.6 & 10.4 & Continuous & 41 & 1.1 & 11.8 & 104 & 0.6 & Transient expansion, regression \\
\hline 13 & 4.0 & 10.5 & Continuous & 49 & 6.7 & 11.1 & 104 & 1.7 & Regression \\
\hline 14 & 3.2 & 10.4 & Continuous & 30 & 6.5 & 11.1 & 109 & 1.8 & Regression \\
\hline 15 & 0.4 & 12.3 & Regrowth & 63 & 2.5 & 11.3 & 109 & 0.3 & Regression \\
\hline 16 & 1.0 & 10.8 & Continuous & 35 & 2.4 & 9.8 & 129 & 1.5 & Regression \\
\hline 17 & 3.6 & 11.6 & Regrowth & 65 & 2.3 & 12.4 & 25 & 2.2 & Transient expansion, stable \\
\hline 18 & 0.60 & 11.2 & Regrowth & 70 & 1.1 & 12.2 & 31 & 1.5 & Transient expansion \\
\hline 19 & 2.4 & 11.2 & Continuous & 37 & 5.3 & 11.8 & 31 & 4.6 & Transient expansion, regression \\
\hline 20 & 0.19 & 10.9 & Continuous & 65 & 0.65 & 12.2 & 24 & 0.26 & Regression \\
\hline 21 & 2.5 & 11.0 & Regrowth & 62 & 3.0 & 11.8 & 33 & 3.0 & Transient expansion, stable \\
\hline 22 & 0.072 & 10.5 & Continuous & 53 & 0.27 & 11.1 & 85 & 0.10 & Transient expansion, regression \\
\hline 23 & 7.8 & 11.3 & Regrowth & 65 & 5.1 & 12.5 & 39 & 9.4 & Transient expansion \\
\hline 24 & 3.2 & 11.0 & Regrowth & 61 & 3.9 & 12.8 & 48 & 1.9 & Transient expansion, regression \\
\hline 25 & 4.6 & 11.1 & Regrowth & 82 & 4.5 & 11.4 & 32 & 6.2 & Enlargement, stable \\
\hline 26 & 1.1 & 11.3 & Continuous & 62 & 2.1 & 12.2 & 21 & 2.2 & Stable \\
\hline 27 & 6.1 & 11.1 & Regrowth & 86 & 3.6 & 12.0 & 20 & 0.58 & Regression \\
\hline 28 & 1.2 & 11.2 & Continuous & 49 & 2.3 & 12.4 & 13 & 2.4 & Stable \\
\hline
\end{tabular}

FU = follow-up in months after GKRS 2; INT = interval in months between both GKRS treatments; MD1 = margin dose in grays delivered to $100 \%$ of tumor volume at GKRS $1 ;$ MD2 = margin dose in grays delivered to $100 \%$ of tumor volume at GKRS 2; TV1 = tumor volume in milliliters at GKRS 1; TV2 = tumor volume in milliliters at GKRS 2; TV3 = tumor volume in milliliters at last follow-up.

* Tumor growth pattern after GKRS 1 and before GKRS 2.

† Tumor growth pattern after GKRS 2 during follow-up.

lyze possible predictors of facial nerve complications after retreatment because of the small number of cases.

\section{Literature Search}

Several series with patients undergoing GKRS retreat- ment have been reported. The query yielded 28 results, and 6 articles were deemed relevant for inclusion after applying our inclusion criteria. ${ }^{3,5,8,11,13,23}$ A summary of the treatment outcomes and complications after GKRS retreatment is shown in Table 7.

TABLE 3. Neurological outcomes in Group 1

\begin{tabular}{|c|c|c|c|c|c|}
\hline \multirow{2}{*}{$\begin{array}{c}\mathrm{HB} \\
\text { Classification }\end{array}$} & \multicolumn{3}{|c|}{ Facial Nerve Function } & \multirow{2}{*}{$\begin{array}{l}\text { Facial } \\
\text { Spasm }\end{array}$} & \multirow{2}{*}{$\begin{array}{l}\text { Trigeminal Nerve } \\
\text { Hypesthesia }\end{array}$} \\
\hline & Before Re-treat & After Re-treat & Last Follow-Up & & \\
\hline I & 28 & 24 & 26 & \multirow{4}{*}{$\begin{array}{l}5 \text { permanent, } 4 \text { transient, } \\
1 \text { unchanged }\end{array}$} & \multirow{4}{*}{$\begin{array}{l}2 \text { permanent, } 2 \text { transient, } \\
2 \text { unchanged }\end{array}$} \\
\hline ॥ & 0 & 4 & 2 & & \\
\hline III & 0 & 0 & 0 & & \\
\hline IV & 0 & 0 & 0 & & \\
\hline
\end{tabular}

$\mathrm{HB}=$ House-Brackmann; Re-treat $=$ retreatment

All data are shown as the number of patients. 
TABLE 4. Patient characteristics in Group 2

\begin{tabular}{|c|c|}
\hline Characteristic & Value \\
\hline No. of patients & 10 \\
\hline Mean age (range) at GKRS 2, yrs & $63(36-82)$ \\
\hline \multicolumn{2}{|l|}{ Sex } \\
\hline Male & 7 \\
\hline Female & 3 \\
\hline \multicolumn{2}{|l|}{ Side of the body } \\
\hline Right & 7 \\
\hline Left & 3 \\
\hline Median interval btwn treatments (range), mos & $35(13-58)$ \\
\hline Median follow-up (range), mos & $94(54-129)$ \\
\hline Median margin dose for GKRS 1 (range), Gy & $11.1(10.1-11.5)$ \\
\hline Median margin dose for GKRS 2 (range), Gy & $11.3(10.0-12.4)$ \\
\hline Median tumor volume at GKRS 1 (range), $\mathrm{cm}^{3}$ & $1.6(0.2-3.3)$ \\
\hline Median tumor volume at GKRS 2 (range), $\mathrm{cm}^{3}$ & $3.9(0.4-9.7)$ \\
\hline Median tumor volume at last follow-up (range), $\mathrm{cm}^{3}$ & $2.1(0.1-5.2)$ \\
\hline \multicolumn{2}{|l|}{ Growth pattern after GKRS 1} \\
\hline Continuous & 8 \\
\hline Regrowth & 1 \\
\hline Regression & 1 \\
\hline Transient expansion after GKRS 2 & 2 \\
\hline \multicolumn{2}{|l|}{ Retreatment result at last follow-up } \\
\hline Regression & 9 \\
\hline Stable & 1 \\
\hline
\end{tabular}

\section{Discussion}

GKRS is a viable primary treatment option for vestibular schwannoma with good long-term tumor control, good quality of life, and low complication rates. GKRS is also a valid secondary treatment after planned subtotal resection. ${ }^{10,17}$ In the case of treatment failure-either because of continuous growth or regrowth of the tumor after the initial response-additional treatment is usually necessary. Whether this second procedure should be microsurgery or radiosurgery remains debatable. ${ }^{16,18,19}$ Scientific evidence that proves the superiority of one treatment modality over the other is currently lacking, and only retrospective observational studies have been reported. The inherent selection bias and lack of uniformity in the outcome criteria of such studies excludes a valid comparison of both treatment modalities. As a result, there is no consensus regarding how to re-treat vestibular schwannomas that have failed to respond to radiosurgery.

Several earlier reports of small patients series showed good tumor control with limited neurological complications after a second radiosurgical procedure. ${ }^{3,5,8,23}$ Liscak et al. achieved tumor control in 22 of 24 patients $(92 \%)$ who underwent a second radiosurgical procedure with a median follow-up of 43 months. ${ }^{11}$ Five patients with treatment failure after the first GKRS treatment underwent microsurgical resection, with none having any remaining facial nerve function after the surgical procedure. Lonneville et al. analyzed 27 retreatment procedures performed in 25 patients with a median follow-up of 46 months and reported a tumor control rate of $85 \%$ with no new neurological symptoms. ${ }^{13}$ Only the growing part of the tumor was treated in 6 patients, which included 2 patients who underwent 3 radiosurgical procedures. In our series of 38 patients with the longest published follow-up period after the second radiosurgical procedure, we observed $100 \%$ tumor control. This is in line with the high tumor control rate observed in previous series, ${ }^{3,5,8,11,13,23}$ Earlier series did not differentiate between patients who did or did not undergo subtotal resection prior to radiosurgery. However, resected tumors are generally larger and might reflect a different biological tumor behavior. Moreover, target definition in postresection cases is more difficult and might influence radiosurgical outcome. Our volumetric analysis shows that retreatment with GKRS is effective in both groups.

While all patients treated with GKRS 2 at our institute

TABLE 5. Tumor volumetric responses of Group 2

\begin{tabular}{cccccccccl}
\hline Case No. & TV1 & MD1 & Growth Pattern $1^{*}$ & INT & TV2 & MD2 & FU & TV3 & Growth Pattern 2 $\uparrow$ \\
\hline 1 & 1.6 & 10.8 & Continuous & 13 & 5.5 & 11.3 & 75 & 5.2 & Stable \\
\hline 2 & 0.9 & 10.1 & Regrowth & 51 & 2.3 & 11.1 & 85 & 1.0 & Regression \\
\hline 3 & 3.3 & 10.8 & Continuous & 42 & 9.6 & 10.0 & 125 & 2.2 & Transient expansion, regression \\
\hline 4 & 3.2 & 10.4 & Continuous & 53 & 6.6 & 12.4 & 54 & 3.3 & Transient expansion, regression \\
\hline 5 & 1.6 & 11.2 & Continuous & 28 & 3.6 & 11.4 & 99 & 0.9 & Regression \\
\hline 6 & 1.5 & 11.2 & Continuous & 27 & 3.7 & 11.2 & 105 & 1.9 & Regression \\
\hline 7 & 0.2 & 11.4 & Continuous & 36 & 0.6 & 10.5 & 129 & 0.1 & Regression \\
\hline 8 & 1.9 & 11.5 & Continuous & 33 & 4.7 & 11.4 & 111 & 2.2 & Regression \\
\hline 9 & 1.5 & 11.3 & Continuous & 30 & 4.0 & 11.6 & 89 & 2.6 & Regression \\
\hline 10 & 0.5 & 10.9 & Regression & 58 & 0.4 & 11.2 & 89 & 0.2 & Regression \\
\hline
\end{tabular}

FU = follow-up in months after GKRS 2; INT = interval in months between both GKRS treatments; MD1 = margin dose in grays delivered to $100 \%$ of tumor volume at GKRS 1; MD2 = margin dose in grays delivered to $100 \%$ of tumor volume at GKRS 2; TV1 = tumor volume in milliliters at GKRS 1; TV2 = tumor volume in milliliters at GKRS 2; TV3 = tumor volume in milliliters at last follow-up.

* Tumor growth pattern after GKRS 1 and before GKRS 2.

$\dagger$ Tumor growth pattern after GKRS 2 during follow-up. 
TABLE 6. Neurological outcomes in Group 2

\begin{tabular}{|c|c|c|c|c|c|}
\hline \multirow{2}{*}{$\begin{array}{c}\mathrm{HB} \\
\text { Classification }\end{array}$} & \multicolumn{3}{|c|}{ Facial Nerve Function } & \multirow{2}{*}{$\begin{array}{l}\text { Facial } \\
\text { Spasms }\end{array}$} & \multirow{2}{*}{$\begin{array}{l}\text { Trigeminal Nerve } \\
\text { Hypesthesia }\end{array}$} \\
\hline & Before Re-treat & After Re-treat & Last Follow-Up & & \\
\hline 1 & 3 & 2 & 2 & \multirow{5}{*}{1 unchanged } & \multirow{5}{*}{$\begin{array}{l}1 \text { permanent, } 1 \text { transient, } \\
1 \text { unchanged }\end{array}$} \\
\hline II & 3 & 2 & 4 & & \\
\hline III & 0 & 1 & 0 & & \\
\hline IV & 0 & 1 & 0 & & \\
\hline Not specified & $4^{*}$ & 4 & 4 & & \\
\hline
\end{tabular}

showed tumor control with an acceptable length of followup, GKRS retreatment is not without risk. We observed a higher percentage of neurological side effects after retreatment. Facial nerve dysfunction was reported in 4 of 420 patients $(0.95 \%)$ after the first radiosurgical procedure at our Gamma Knife center. ${ }^{9}$ Using the same treatment protocol and treatment indications, 3 of 38 patients (7.9\%) developed (seemingly) permanent House-Brackmann Grade II facial nerve dysfunction after GKRS 2. Persisting trigeminal nerve dysfunction might also occur more frequently after retreatment ( 3 of 38 patients after GKRS 2 [7.9\%] vs 13 of 420 patients after GKRS 1 [3.1\%]), as well as persisting facial spasms. ${ }^{9}$ These side effects are nevertheless relatively mild and infrequent compared with the alternative of microsurgery after radiosurgery, which is associated with worse functional outcome in cranial nerve function preservation and additional surgical morbidity., ${ }^{4,18}$ Extensive scarring and tumor adhesion after radiosurgery can complicate matters further, limiting surgical resection to subtotal or only partial tumor removal.,20,22 The increase in neurological side effects after GKRS 2 observed in this study might be an argument for considering hypofractionated stereotactic radiotherapy as a second procedure be- cause theoretically hypofractionation might be less harmful to the surrounding critical structures, like the facial nerve and possibly the trigeminal nerve, within the target volume. Hypofractionated stereotactic radiotherapy has shown comparable tumor control to single-fraction stereotactic radiotherapy. ${ }^{2,12}$ However, a recent review by Apicella et al. did not show a significant difference in hearing preservation or neurological complications after GKRS or fractionated radiotherapy. ${ }^{1}$ To the best of our knowledge, no series of hypofractionated stereotactic radiotherapy after failed GKRS has been reported.

One of the reasons for our volumetric analysis is the hesitation to re-treat patients who do not show any response after the first procedure. Assuming a reproducible radiobiological effect, one would expect a similar volumetric effect after GKRS 1 and GKRS 2. Consequently, the choice for GKRS 2 might appear more logical when GKRS 1 results in longer lasting tumor shrinkage, as opposed to no response and continued growth. Our analysis did not show such reproducibility. With the observed high level of tumor control, this justifies GKRS 2, even in the cases when no volumetric effect is observed after GKRS 1. The mechanism underlying the observed high tumor

TABLE 7. Literature overview of outcomes after repeat GKRS

\begin{tabular}{|c|c|c|c|c|c|c|c|c|c|}
\hline $\begin{array}{l}\text { Authors } \\
\& \text { Year }\end{array}$ & $\begin{array}{l}\text { No. of } \\
\text { Patients }\end{array}$ & MD & $\begin{array}{c}\text { TV } \\
\text { (range) }\end{array}$ & INT & FU & Tumor Outcome & $\begin{array}{l}\text { Facial Nerve } \\
\text { Complication }\end{array}$ & $\begin{array}{l}\text { Trigeminal Nerve } \\
\text { Complication }\end{array}$ & $\begin{array}{c}\text { Adverse } \\
\text { Radiation Effect }\end{array}$ \\
\hline $\begin{array}{l}\text { Hasegawa et al., } \\
2005\end{array}$ & 4 & - & - & 30 & - & $\begin{array}{l}3 \text { regression, } 1 \text { salvage } \\
\text { surgery }\end{array}$ & None & None & None \\
\hline $\begin{array}{l}\text { Dewan \& Norén, } \\
2008\end{array}$ & 11 & 12.0 & $2.1(1.4-3.3)$ & 51 & - & $\begin{array}{l}8 \text { regression, } 1 \text { stabilization, } \\
2 \text { enlargement }\end{array}$ & None & $\begin{array}{c}2 \text { increased facial } \\
\text { numbness }\end{array}$ & $\begin{array}{r}1 \text { symptomatic, } 1 \\
\text { asymptomatic }\end{array}$ \\
\hline Yomo et al., 2009 & 8 & 12.0 & $1.3(0.5-3.1)$ & 46 & 64 & 6 regression, 2 stabilization & None & None & None \\
\hline Kano et al., 2010 & 6 & 11.5 & $2.1(0.9-6.4)$ & 63 & 29 & 4 regression, 2 stabilization & None & None & 1 asymptomatic \\
\hline $\begin{array}{l}\text { Lonneville et al., } \\
2015\end{array}$ & $25^{\star}$ & 12.0 & $2.3(0.2-8.3)$ & 45 & 46 & $\begin{array}{l}15 \text { regression, } 8 \text { stabilization, } \\
4 \text { enlargement }\end{array}$ & None & None & None \\
\hline Present study & 38 & 11.5 & $3.6(0.3-9.4)$ & 49 & 75 & $\begin{array}{l}28 \text { regression, } 6 \text { stabilization, } \\
2 \text { transient expansion, } 2 \\
\text { enlargement (stable) }\end{array}$ & $\begin{array}{l}5 \text { spasms, } \\
3 \text { palsy } \\
\text { (HB II) }\end{array}$ & 3 hypesthesia & 1 symptomatic \\
\hline
\end{tabular}

FU = follow-up in months after GKRS 2; INT = interval in months between both GKRS treatments; $\mathrm{MD}=$ margin dose in grays delivered to $100 \%$ of tumor volume; TV = tumor volume in milliliters.

* Two patients underwent 3 GKRS procedures. 
control rates after the second procedure is unclear. One might argue that the radiation delivered during GKRS 1 was insufficient because of either inadequate target definition or dose planning. We find this explanation unlikely because target definition was done by the neurosurgeon and checked by the radiation oncologist. Dose prescription is performed using a highly uniform protocol. More likely, the high efficacy of GKRS 2 is explained by the cumulative radiobiological effects of both procedures.

Given the high rate of tumor control observed at our institution, we routinely recommend GKRS 2 after the failure of GKRS 1 in patients with a small- to medium-size vestibular schwannoma. Transient radiation-induced tumor swelling, which has been reported to be highest at 6 to 12 months after GKRS and stabilizing after 12 to 36 months, must be taken into consideration. ${ }^{6,14}$ Therefore, GKRS retreatment should, in our opinion, only be considered after a minimum follow-up period of 24 months. Over the years, we have observed transient tumor swelling beyond this point. Consequently, we nowadays observe enlarged tumors longer before the decision to re-treat is made.

\section{Study Limitations}

The retrospective nature of this study is a limitation. The patients in this study were treated over a period of 14 years, and although we attempted to describe our workup and treatment decisions as clearly as possible, it is unlikely that we would make the exact same decisions now that we made a decade ago. The indication for microsurgery as a second intervention after failed GKRS has certainly changed during this period, and we have become more liberal in accepting larger vestibular schwannomas for GKRS 2. Also, there has been a tendency to apply a wait-and-see policy over longer periods of time when increased tumor volume is observed during the follow-up but before classification as radiosurgical treatment failure. Although we are certain that cranial nerve function was adequately assessed during follow-up, the lack of available audiograms means that hearing preservation was not analyzed in this study.

As a tertiary referral center, we have treated a substantial number of patients who had their tumors resected at other centers before being referred to us for a radiosurgical procedure on the tumor remnant. The postsurgical cases described in this study are therefore a heterogeneous group because the primary resection indication-the intention to leave the tumor remnant and the tendency to wait for the remnant to grow-varied.

\section{Conclusions}

Repeat GKRS procedure after GKRS 1 has failed appears to be an effective strategy in terms of tumor control. There seems to be an increased risk of mild facial and trigeminal nerve dysfunction in comparison with GKRS 1 , but this risk is acceptable compared with the alternative of microsurgical resection. The volumetric response after GKRS 2 was not consistent with the volumetric response observed after GKRS 1 . This justifies considering GKRS 2 even for tumors that do not show any volumetric response after the first radiosurgical procedure.

\section{References}

1. Apicella G, Paolini M, Deantonio L, Masini L, Krengli M: Radiotherapy for vestibular schwannoma: review of recent literature results. Rep Pract Oncol Radiother 21:399-406, 2016

2. Champ CE, Shen X, Shi W, Mayekar SU, Chapman $\mathrm{K}$, Werner-Wasik M, et al: Reduced-dose fractionated stereotactic radiotherapy for acoustic neuromas: maintenance of tumor control with improved hearing preservation. Neurosurgery 73:489-496, 2013

3. Dewan S, Norén G: Retreatment of vestibular schwannomas with Gamma Knife surgery. J Neurosurg 109 Suppl:144-148, 2008

4. Gerganov VM, Giordano M, Samii A, Samii M: Surgical treatment of patients with vestibular schwannomas after failed previous radiosurgery. J Neurosurg 116:713-720, 2012

5. Hasegawa T, Kida Y, Kobayashi T, Yoshimoto M, Mori Y, Yoshida J: Long-term outcomes in patients with vestibular schwannomas treated using gamma knife surgery: 10-year follow up. J Neurosurg 102:10-16, 2005

6. Hasegawa T, Kida Y, Yoshimoto M, Koike J, Goto $\mathrm{K}$ : Evaluation of tumor expansion after stereotactic radiosurgery in patients harboring vestibular schwannomas. Neurosurgery 58:1119-1128, 2006

7. Iwai Y, Ishibashi K, Nakanishi Y, Onishi Y, Nishijima $\mathrm{S}$, Yamanaka $\mathrm{K}$ : functional outcomes of salvage surgery for vestibular schwannomas after failed Gamma Knife radiosurgery. World Neurosurg 90:385-390, 2016

8. Kano H, Kondziolka D, Niranjan A, Flannery TJ, Flickinger JC, Lunsford LD: Repeat stereotactic radiosurgery for acoustic neuromas. Int J Radiat Oncol Biol Phys 76:520-527, 2010

9. Klijn S, Verheul JB, Beute GN, Leenstra S, Mulder JJ, Kunst HP, et al: Gamma Knife radiosurgery for vestibular schwannomas: evaluation of tumor control and its predictors in a large patient cohort in The Netherlands. J Neurosurg 124:1619-1626, 2016

10. Kondziolka D, Lunsford LD, McLaughlin MR, Flickinger JC: Long-term outcomes after radiosurgery for acoustic neuromas. N Engl J Med 339:1426-1433, 1998

11. Liscak R, Vladyka V, Urgosik D, Simonova G, Vymazal J: Repeated treatment of vestibular schwannomas after gamma knife radiosurgery. Acta Neurochir (Wien) 151:317-324, 2009

12. Litre F, Rousseaux P, Jovenin N, Bazin A, Peruzzi P, Wdowczyk D, et al: Fractionated stereotactic radiotherapy for acoustic neuromas: a prospective monocenter study of about 158 cases. Radiother Oncol 106:169-174, 2013

13. Lonneville S, Delbrouck C, Renier C, Devriendt D, Massager N: Repeat Gamma Knife surgery for vestibular schwannomas. Surg Neurol Int 6:153, 2015

14. Nagano O, Higuchi Y, Serizawa T, Ono J, Matsuda S, Yamakami I, et al: Transient expansion of vestibular schwannoma following stereotactic radiosurgery. J Neurosurg 109:811-816, 2008

15. Pollock BE, Driscoll CL, Foote RL, Link MJ, Gorman DA, Bauch CD, et al: Patient outcomes after vestibular schwannoma management: a prospective comparison of microsurgical resection and stereotactic radiosurgery. Neurosurgery 59:77-85, 2006

16. Pollock BE, Link MJ: Vestibular schwannoma radiosurgery after previous surgical resection or stereotactic radiosurgery. Prog Neurol Surg 21:163-168, 2008

17. Pollock BE, Lunsford LD, Flickinger JC, Clyde BL, Kondziolka D: Vestibular schwannoma management. Part I. Failed microsurgery and the role of delayed stereotactic radiosurgery. J Neurosurg 89:944-948, 1998

18. Pollock BE, Lunsford LD, Kondziolka D, Sekula R, Subach BR, Foote RL, et al: Vestibular schwannoma management: 
Part II. Failed radiosurgery and the role of delayed microsurgery. J Neurosurg 119 Suppl:949-955, 2013

19. Roche PH, Khalil M, Thomassin JM, Delsanti C, Régis J: Surgical removal of vestibular schwannoma after failed Gamma Knife radiosurgery. Prog Neurol Surg 21:152-157, 2008

20. Slattery WH III, Brackmann DE: Results of surgery following stereotactic irradiation for acoustic neuromas. Am J Otol 16:315-321, 1995

21. van de Langenberg R, Hanssens PE, van Overbeeke JJ, Verheul JB, Nelemans PJ, de Bondt BJ, et al: Management of large vestibular schwannoma. Part I. Planned subtotal resection followed by Gamma Knife surgery: radiological and clinical aspects. J Neurosurg 115:875-884, 2011

22. Wise SC, Carlson ML, Tveiten OV, Driscoll CL, Myrseth E, Lund-Johansen M, et al: Surgical salvage of recurrent vestibular schwannoma following prior stereotactic radiosurgery. Laryngoscope 126:2580-2586, 2016

23. Yomo S, Arkha Y, Delsanti C, Roche PH, Thomassin JM, Régis J: Repeat Gamma Knife surgery for regrowth of vestibular schwannomas. Neurosurgery 64:48-55, 2009

\section{Disclosures}

The authors report no conflict of interest concerning the materi- als or methods used in this study or the findings specified in this paper.

\section{Author Contributions}

Conception and design: Verheul, Fu. Acquisition of data: Fu, Kunst, Mulder, Hanssens. Analysis and interpretation of data: Verheul, Fu, Hanssens. Drafting the article: Verheul, Fu. Critically revising the article: all authors. Reviewed submitted version of manuscript: all authors. Approved the final version of the manuscript on behalf of all authors: Verheul. Statistical analysis: Fu. Study supervision: Verheul, Beute, Leenstra, Hanssens.

\section{Supplemental Information}

Previous Presentations

Portions of this manuscript were presented as an oral presentation at the 12th International Stereotactic Radiosurgery Society Congress, June 7-11, 2015, in Yokohama, Japan.

\section{Correspondence}

Jeroen B. Verheul, Gamma Knife Center, St. Elisabeth Hospital, PO Box 90151, Tilburg, 5000 LC, The Netherlands. email: j.verheul@etz.nl. 